\title{
Acurácia e tempos de resposta de máquinas para aplicação de defensivos agrícolas à taxa variável
}

\author{
Accuracy and response times of variable rate machines for agricultural defensive application
}

\author{
Otávio Dias da Costa Machado I, II Airton dos Santos Alonço ${ }^{\mathrm{I}}$ Tiago Rodrigo Francetto ${ }^{\mathrm{I}}$ \\ Dauto Pivetta Carpes ${ }^{\mathrm{I}}$
}

\section{- REVISÃO BIBLIOGRÁFICA -}

\section{RESUMO}

$O$ desenvolvimento da agricultura de precisão $e$ do manejo localizado teve como premissa a atuação precisa das máquinas agrícolas. Entretanto, ainda não está disseminado o conhecimento sobre a qualidade operacional dessas máquinas. A partir disso, objetivou-se a descrição dos parâmetros de desempenho dessas máquinas, bem como as variáveis que os caracterizam e os métodos de ensaio utilizados. Foi possível verificar que muitos dos equipamentos estão operando com tempos de resposta excessivos para suas aplicações, com baixa acurácia e distorções na dosagem. Em alguns casos, foi possivel identificar que as máquinas tiveram precisão adequada. Dessa forma, os resultados discutidos podem informar aspectos importantes para a seleção e uso das máquinas, para que atendam no campo ao manejo planejado.

Palavras-chave: agricultura de precisão, sistemas de controle, dosagem de insumos.

\section{ABSTRACT}

The development of precision agriculture and sitespecific management were premised on farm machinery precise actuation. However, this performance is still unacquainted. Along this, it was established the aim of describing the performance of this machines, the variables evolved and the applied methods. It was demonstrated that many of the devices are operating with excessive response times, low accuracy and distorted measures. In some cases, it was possible to identify situations with appropriate precision. Thus, it could notice important aspects to be considered, to make a field work indeed to the planed management.

Key words: precision agriculture, control systems, input rate.

\section{INTRODUÇÃO}

A Agricultura de Precisão (AP) moderna desenvolveu-se como um novo sistema de produção agrícola, com o advento do geo-referenciamento dos atributos dos fatores de produção. Em seus primórdios, segundo MOLIN (1997), o embasamento do sistema estava na análise da variabilidade espacial dos fatores de produção, especialmente do solo. Essa análise permitia então a tomada de decisões para a aplicação dos insumos. Já nos últimos 20 anos, segundo ANDRADE-SANCHEZ \& HEUN (2010), o estudo da variabilidade e a adequação das intervenções evoluíram como um novo paradigma, o denominado manejo localizado. Os autores o descreveram como aquele que utiliza intensamente energia e insumos, o qual é empregado nas dosagens adequadas, no tempo certo e no lugar exato.

Entretanto, nem os modernos sistemas de localização, nem os recursos computacionais que operacionalizam o manejo localizado terão sucesso se as máquinas para aplicação à Taxa Variável (TV) não corresponderem, no campo, ao planejamento. MOLIN (1997) já observava este fato, pois afirmou que as intervenções deveriam ocorrer de forma localizada e com dosagens precisas. Atualmente, com base na expansão do mercado de AP e TV, tanto no número de equipamentos quanto nos tipos de operações agrícolas

'Laboratório de Pesquisa e Desenvolvimento de Máquinas Agrícolas (Laserg), Universidade Federal de Santa Maria (UFSM), Santa Maria, RS, Brasil.

"Instituto Federal de Educação Ciência e Tecnologia do Rio Grande do Sul (IFRS), Campus Bento Gonçalves, Avenida Osvaldo Aranha, 540, 95700-000, Bento Gonçalves, RS, Brasil. E-mail: otavio.machado@bento.ifrs.edu.br. Autor para correspondência. 
realizadas, as máquinas estão disseminando-se no campo mais rapidamente que a evolução da pesquisa, o que faz necessário reconhecer os potenciais dos novos recursos, em especial dos sistemas de controle.

A partir disso, foi realizada uma compilação de dados para a identificação dos desempenhos dos sistemas de controle, a descrição de suas avaliações e do nível de desempenho dos tempos de resposta e da acurácia das máquinas.

Avaliação de desempenho de sistemas de controle

O desempenho dos sistemas de controle é medido pela qualidade da execução do acionamento que realizam, os quais incluem desde direcionamento de tratores, acionar e desligar elementos das máquinas e controlar a taxa de descarga ou dosagem de insumos. A dosagem é a mais importante, resultado da combinação de fatores do sistema, da máquina e do insumo aplicado. Ela é avaliada pelos tempos de resposta e pela acurácia, nos regimes de acionamento transitório e estacionário, respectivamente. $\mathrm{O}$ primeiro é caracterizado pela mudança de dosagem, em forma de degraus ou rampas, pois representa a passagem de uma dosagem para outra, enquanto o segundo ocorre com dosagens constantes, quando não estão ocorrendo variações. Regimes transitórios irão ocorrer nas mudanças de zonas, para máquinas que utilizam mapas; nas mudanças de leituras de sensores, as quais alteram a prescrição em tempo real; nas alterações de dose comandadas pelo operador em sistemas de malha aberta, ou mesmo nas mudanças de velocidade.

As alterações de dosagem ocorrem com atrasos em relação ao comando. Segundo OGATA (2011), a alteração da dosagem mediante uma excitação do tipo degrau representa o fenômeno de resposta do regime transitório, sendo apresentado na figura 1. Nos primeiros momentos, a alteração da dosagem inicia-se lentamente, seguida de uma fase mais rápida, verificada pela maior inflexão da curva, que segue esta tendência até atingir a dosagem final. A partir desse momento, o sistema entra em uma acomodação próxima da prescrição final.

Cada etapa da transição corresponde a uma variável do estudo do fenômeno de resposta do sistema de controle. A primeira ocorre quando a curva de resposta atinge metade do valor final pela primeira vez, denominada tempo de atraso $\left(t_{a t}\right)$. Após essa etapa, o sistema irá atingir o denominado tempo de subida $\left(t_{s}\right)$, também denominado tempo de transição, caracterizado pela mudança do sistema de 10 para $90 \%$ do valor final. O tempo de subida também pode ser avaliado nos intervalos de 5 a $95 \%$ ou de zero a 100\% (OGATA, 2011).
Após o sistema atingir a dosagem meta, ocorre um ponto máximo acima dela, no qual o tempo decorrido denomina-se tempo de pico $\left(t_{p}\right)$. Neste ponto, além do tempo, é avaliado o máximo sobre-sinal $\left(M_{s}\right)$, que é o máximo valor de pico da curva de resposta e indica a estabilidade relativa do sistema. A última variável componente de um fenômeno de resposta é caracterizada quando o sistema atinge valores próximos à meta final, o tempo de acomodação $\left(t_{a c}\right)$. Esses valores são considerados em uma faixa de 2 a $5 \%$ da dosagem ideal, sendo a maior variável de tempo avaliada (OGATA, 2011).

Para o estudo dos fenômenos de resposta do regime transitório, poderão ser utilizados métodos diretos, que aferem o resultado final ou insumo dosado, e métodos indiretos, em que outras variáveis, que não do insumo, substituem essa leitura. Os métodos diretos são mais simples de serem aplicados, pois envolvem, por exemplo, aferições da massa de insumos coletada (FULTON et al., 2001; FULTON et al., 2003) ou a avaliação da população de plantas obtidas em diferentes zonas (FULTON et al., 2006). Já os métodos indiretos, monitoram através de sensores os componentes que realizam a dosagem, tais como a velocidade de esteiras dosadoras (SCHUMANN et al., 2006), rotação do mecanismo dosador (UMEZU, 2003) ou a abertura da comporta de dosagem (CERRI, 2001).

Os métodos diretos têm uma aplicabilidade expressiva para distribuidores, em que a coleta do insumo poderá ocorrer no sentido longitudinal ao deslocamento em linhas laterais de coletores (CERRI, 2001; MOLIN \& MENEGATTI, 2003; SERRANO et al., 2007), permitindo a aferição do tempo de resposta. Entretanto, a distribuição transversal pode ser avaliada concomitantemente, utilizando-se um arranjo de coletores em duas dimensões (FULTON et al., 2001). Já os métodos indiretos têm a facilidade de permitir a execução em ambiente controlado, e não necessitam de todos os componentes da máquina. UMEZU (2003), após desenvolver um sistema de controle de dosadores helicoidais de fertilizantes, comparou as tensões de comando do sistema e as tensões das rotações correspondentes do acionamento.

As avaliações em bancada também podem aplicar a metodologia de degraus múltiplos, ou seja, uma sequência de trocas de prescrição, mais facilmente que no campo, sendo apresentados na figura 2 exemplos em malha aberta e fechada. $\mathrm{Na}$ malha aberta, sem aferição da rotação comandada, observam-se distorções expressivas em relação à resposta ideal. Na malha fechada, as respostas são mais adequadas, mas a dosagem ainda oscila próximo da meta (UMEZU, 2003). 


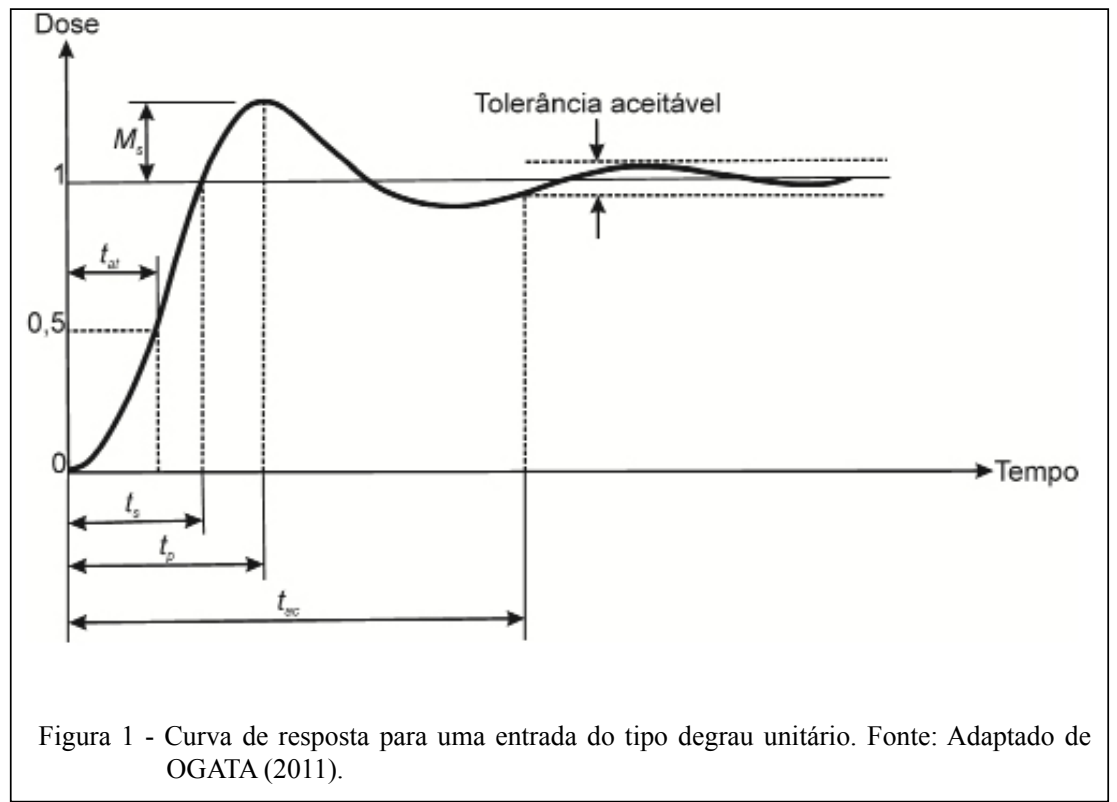

Já no regime estacionário, no qual as dosagens são mantidas homogêneas, não são avaliados tempos de transição entre as dosagens, mas sim a acurácia e a estabilidade do sistema. A acurácia representa um erro porcentual entre a dosagem e a prescrição, enquanto a estabilidade é a variabilidade desta, ambas podendo ser avaliadas de forma direta ou indireta. No desenvolvimento de um controlador para distribuição de calcário, CERRI (2001) utilizou a medição indireta para os testes preliminares da acurácia do equipamento, tendo em vista esta facilitar a etapa de desenvolvimento, pois não necessitava coletar insumo. Nesse caso, a acurácia representava os desvios entre os valores meta, da abertura comandada da comporta de dosagem do equipamento e os valores realmente obtidos. Entretanto, o autor realizou avaliação final do equipamento, com método direto, com aplicação do insumo e coleta em três diferentes zonas do mapa que usou na área de testes.

Metodologias para avaliação de sistemas de controle Para o entendimento do estado da arte do desempenho dos sistemas de controle, primeiramente, é necessário identificar quais normas e procedimentos são adotados nas avaliações, para posteriormente reconhecer as variáveis e o potencial desempenho. Por não existir normas específicas para ensaio de máquinas à TV, SHEARER et al. (2002) elaboraram considerações para uma futura norma para avaliação de controladores, com foco na distribuição de insumos a lanço. Os autores destacaram que deverão ser adaptadas às normas existentes, baseando-se nas normas da American Society of Agricultural Engineers (ASAE), ASAE S341.2, procedimentos para medição da uniformidade de distribuição e calibração de distribuidores a lanço; ASAE EP371.1, procedimento para calibração de aplicadores granulares e ASAE EP367.2, guia para preparação de calibração de pulverizadores. As normas são aplicadas, originalmente, em taxas constantes.

Para os distribuidores, SHEARER et al. (2002) mantiveram a matriz de coletores em duas dimensões, preconizada por FULTON et al. (2001). Entretanto, propuseram o uso de uma segunda matriz, para avaliar o recobrimento em passagens consecutivas. Essas metodologias foram posteriormente aplicadas por FULTON (2003) e FULTON et al. (2005).

A metodologia de avaliação de distribuidores pela coleta de insumos não leva em consideração os sinais de comando emitidos pelo controlador ou mesmo não obtém a resposta da rotação das esteiras ou da abertura longitudinal da comporta do sistema dosador. Somente é aferido o resultado global diretamente pela coleta de insumos. Portanto, é um resultado combinado do desempenho dos componentes do sistema de controle e também do insumo e outros fatores, independentes das máquinas. Com essa metodologia, não é possível dissociar o fenômeno de resposta quanto às influências da latência do fluxo, dos componentes eletrônicos, mecânicos, hidráulicos ou do software.

O trabalho de SHEARER et al. (2002) complementa outros aspectos para as avaliações,

Ciência Rural, v.45, n.3, mar, 2015. 

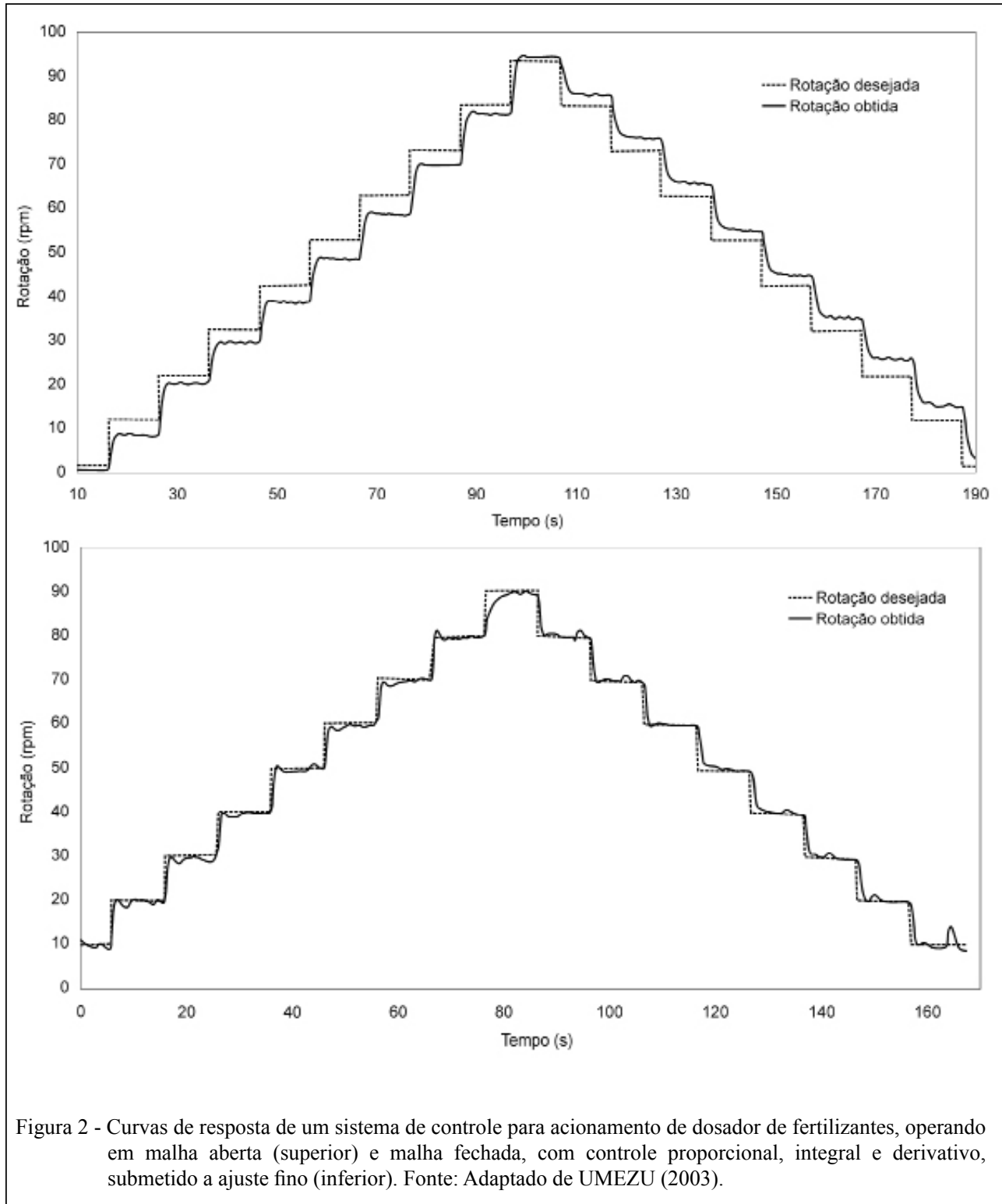

pois eles recomendaram que à norma ASAE S341.2 deve ser adicionado procedimento para avaliação do tempo de atraso no fluxo do material, no equipamento e até o solo, para fins de determinação de tempos de adiantamento e que as configurações e os componentes do controlador devem ser especificados. Os autores determinaram também que, durante os testes, o abastecimento de insumos deve ser representativo da operação de campo, de forma que os dosadores operem em seu torque normal de acionamento e que os sensores, utilizados para aquisição de dados, devem garantir acurácia adequada dos valores coletados nas avaliações. Quanto ao roteiro pelo qual os testes devem ser conduzidos, afirmaram que devem ser realizadas trocas de dosagem e frequências correspondentes à utilização dos equipamentos no campo. Para isso, estabeleceram uma sequência de degraus correspondente às combinações entre as doses de $0,50,75$ e $90 \%$ para as transições.

Havendo poucos recursos metodológicos atualmente para avaliar máquinas à TV, a norma ASAE S341.2 também serviu de referência para GARCIA (2007), ao testar um sistema de controle para dosagem de fertilizantes com dosadores helicoidais. Entretanto, espera-se que seja publicada uma nova norma, pois, segundo ASABE (2012), dentre os projetos que estão tramitando, no grupo PM54: Agricultura de Precisão, há o projeto de norma

Ciência Rural, v.45, n.3, mar, 2015. 
X573: Procedimentos para avaliação de acurácia de aplicação à TV de materiais granulares.

Para desenvolver um protocolo de testes para máquinas à $\mathrm{TV}$, é possível ainda considerar da normaASAE S341.3 (ASABE, 2004) o preenchimento do reservatório de fertilizantes granulares em 40 a $50 \%$ da capacidade e, caso não utilizados no prazo de $4 \mathrm{~h}$, deve ser esvaziado e abastecido novamente. A rotação de acionamento deve ser correspondente à recomendação do fabricante e a velocidade escolhida para o teste deve ser correspondente ao intervalo descrito pelo fabricante e mantida homogênea.

Quanto ao uso dos tempos de resposta, deve-se esclarecer algumas diferenças entre os experimentos. Diferentemente do que OGATA (2011) considerou, na metodologia desenvolvida por FULTON et al. (2001), utilizada por FULTON et al. (2005) e SCHUMANN et al. (2006), o tempo de atraso foi aferido quando o sinal atingiu $10 \%$ da mudança de comando e o tempo de subida, denominado de tempo de transição, foi medido até o comando atingir $90 \%$ da troca. Dessa forma, foi considerado o tempo de resposta pelo somatório do tempo de atraso e tempo de transição. Em outros experimentos, como MOLIN \& MENEGATTI (2003), CERRI (2001) e SERRANO et al. (2007), não foi possível determinar o tempo de atraso, somente foi obtido o tempo de resposta entre zero a $100 \%$ da dosagem.

As metodologias para avaliação da acurácia também podem ser diretas ou indiretas, as quais avaliam a correspondência entre valores prescritos e aplicados, ou prescritos e aferidos por sensores, respectivamente. Segundo SHEARER et al. (2002), a acurácia afere-se na diferença porcentual entre a prescrição que o controlador lê na zona do mapa, ou determina pelo cálculo do seu algoritmo para sistemas em tempo real, denominada taxa pretendida e a taxa de descarga real obtida em cada ponto no campo. A acurácia pode apresentar valores positivos e negativos, sendo analisada em conjunto com suas medidas de dispersão. Sua representação pode ocorrer em gráficos, os quais correlacionam a dosagem prescrita e obtida em diferentes pontos de regulagem da máquina. Normalmente, o valor apresenta distorções, pois não ocorre uma correspondência perfeita entre as metas e as dosagens, utilizando-se o coeficiente de correlação para qualificar a relação entre estas variáveis (FULTON, et al., 2001).

Segundo SHEARER et al. (2002), a acurácia das máquinas pode ser comprometida no desenvolvimento e na montagem do sistema de controle, devido à qualidade do projeto e dos componentes. A deficiência no conhecimento sobre o desempenho dos sistemas de controle poderá gerar ainda pior resultado em casos comuns no mercado norte-americano, quando os agricultores montam um sistema adaptado em uma máquina, a partir de diferentes fornecedores de componentes. Os autores afirmaram que os erros relativos à acurácia são significativos tanto para aplicação de sólidos quanto de líquidos, que têm impacto final na renda dos produtores, sendo desconhecidos pelos usuários. Estes erros podem também ser gerados pelo usuário, durante a calibração e uso da máquina. Os autores comentaram ainda que a qualidade das respostas das máquinas pode ser desconhecida, mesmo pelos fabricantes, assistência técnica e revendendores.

Desempenho de sistemas de controle

Para a descrição do potencial de desempenho das máquinas para TV quanto ao tempo de resposta, foram compilados dados de 10 experimentos, nos quais foram avaliados 17 equipamentos em 18 ensaios distintos, estando os dados dispostos na tabela 1 . O limite de desempenho exigido dos equipamentos pulverizadores, segundo FIGUEIREDO \& ANTUNIASSI (2006), é de 5 segundos (s). Em aplicações de fertilizantes em citros, com distribuidores centrífugos, para ajuste da dosagem ao porte das árvores (singletree), SCHUMANN et al. (2006) afirmaram ser necessário um segundo. UMEZU (2003) considerou adequado, para o sistema de controle de dosadores de fertilizantes, seu equipamento com um tempo de resposta de $4 \mathrm{~s}$, com restrições para a velocidade de trabalho a ser adotada.

O tempo de resposta observado nos ensaios de UMEZU (2003), é maior para a malha fechada, neste caso, 2,6s superior à malha aberta. Esse é um comportamento característico da malha fechada, na qual as correções do sinal atuante do erro de acionamento provocam oscilações, que aumentam o tempo de resposta, entretanto, com maior aproximação dos resultados ideais de dosagem. No trabalho do autor, pode ainda ser verificada a ocorrência de menores tempos na fase decrescente do regime transitório. Esta última afirmativa foi corroborada por GARCIA (2007), para um sistema semelhante, o qual teve desempenho mais rápido que $\mathrm{o}$ anterior, sendo validado pelo autor.

Dentre os equipamentos avaliados pelos autores consultados, para os 6 pulverizadores apenas dois equipamentos desempenharam tempos menores que $5 \mathrm{~s}$, demonstrando que, nessa operação, as transições podem estar sendo muito lentas e inadequadas para os sistemas de TV. Percebeu-se 
Tabela 1 - Tempos de resposta de máquinas com aplicação à taxa variável.

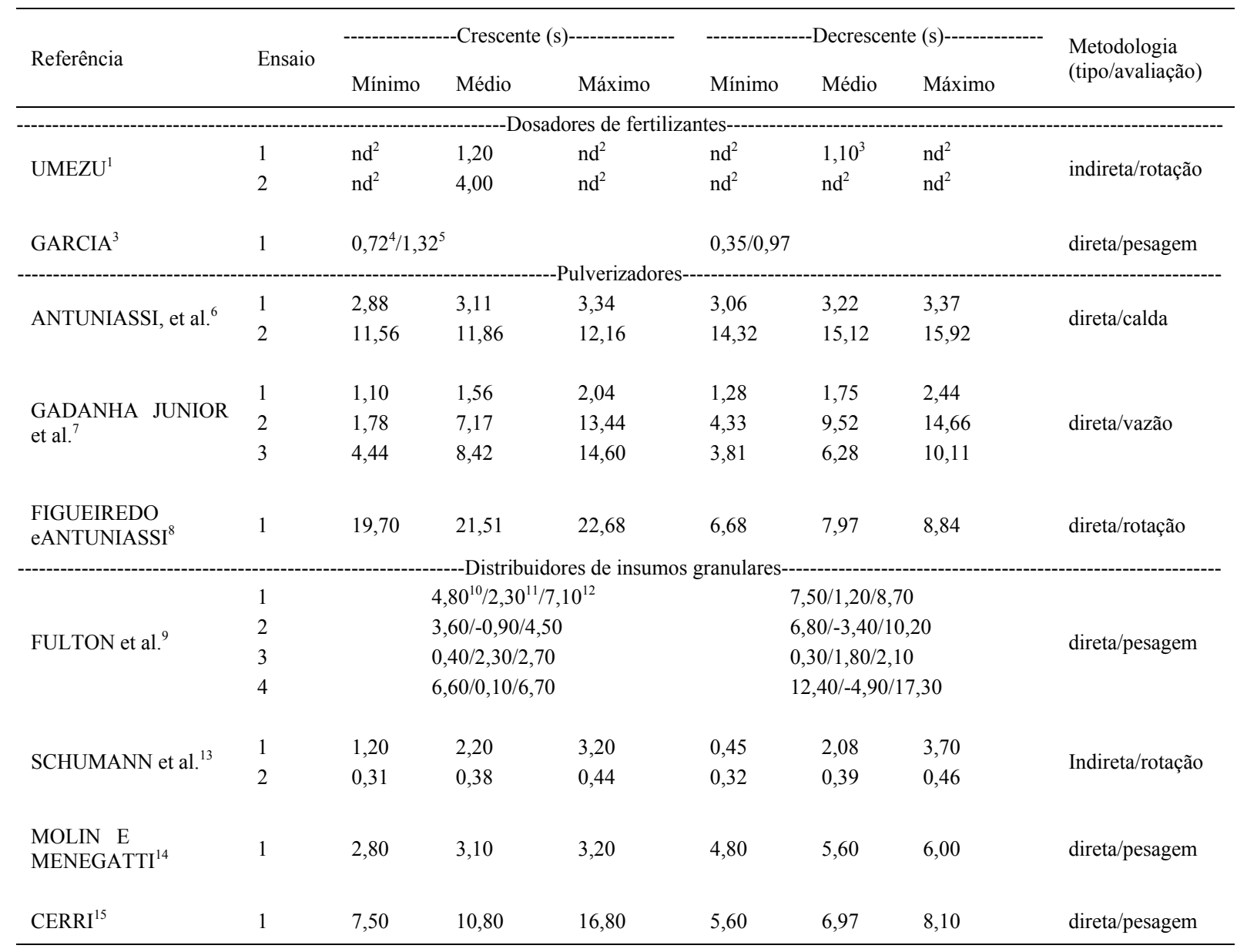

${ }^{1}$ UMEZU (2003): Dosador helicoidal, testes degraus múltiplos. Ensaios: 1) malha aberta; 2) malha fechada.

${ }^{2}$ Dados não disponíveis ou não estimáveis, pelas informações divulgadas.

${ }^{3}$ GARCIA (2007): Dosador helicoidal, dosagens correspondentes às velocidades do dosador de $0,5-2,5-0,5 \mathrm{~m} \mathrm{~s}^{-1}$.

${ }^{4}$ tempo de atraso $/{ }^{5}$ tempo de transição.

${ }^{6}$ ANTUNIASSI, et al. (2002): Sistemas para injeção direta de defensivos. Dosagens dos ensaios: Sistema 1) 1,50-4,50-1,50L min ${ }^{-1}$. Sistema 2) $0,30-0,90-0,30 \mathrm{~L} \mathrm{~min}^{-1}$.

${ }^{7}$ GADANHA JUNIOR et al. (2001): Sistema para controle da vazão. Dados médios das vazões 9,2; 18,2 e 36,4L min ${ }^{-1}$ e dos degraus de velocidade 4-6-4; 4-8-4 e 6-8-6 $\mathrm{km} \mathrm{h}^{-1}$, para 3 diferentes controladores (ensaios 1, 2 e 3).

${ }^{8}$ FIGUEIREDO E ANTUNIASSI (2006): Controle de rotação da bomba. Dados médios para 3 rampas unitárias.

${ }^{9}$ FULTON et al. (2005): Equipamentos centrífugos (1 e 2) e pneumáticos (3 e 4); somente dados médios nas dosagens dos ensaios: 1 ) 56-168 e $168-56 \mathrm{~kg} \mathrm{ha}^{-1}$; 2,3 e 4) $112-336$ e $336-112 \mathrm{~kg} \mathrm{ha}^{-1}$.

${ }^{10}$ Tempo de transição $/{ }^{11}$ Tempo de atraso $/{ }^{12}$ Tempo de resposta.

${ }^{13}$ SCHUMANN et al. (2006): Equipamento centrífugo com dois sistemas de controle (ensaios 1 e 2), médias para dosagens 0-560 e 280-560 e para $560-0$ e $560-280 \mathrm{~kg} \mathrm{ha}^{-1}$.

${ }^{14}$ MOLIN E MENEGATTI (2003): Equipamento centrífugo, dois discos. Médias para dosagens $50-100$ e $50-150$ e para $100-50$ e $150-50 \mathrm{~kg}$ ha $^{-1}$ de ureia.

${ }^{5}$ CERRI (2001): Equipamento centrífugo, dois discos, arrasto. Médias para dosagens 2-3; 2-5; 3-5 Mg ha ${ }^{-1}$ e para 3-2; 5-2 e 5-3Mg ha ${ }^{-1}$ de calcário.

também contrastes entre equipamentos comerciais para a mesma aplicação, tendo em vista que o sistema de injeção direta de defensivos apresentado por ANTUNIASSI et al. (2002), no ensaio 1, apresentou tempos de resposta médios de 3,11 e 3,22s nas fases crescente e decrescente da troca de dosagem, enquanto os demais apresentaram tempos excessivos. Situação semelhante foi observada em GADANHA JUNIOR et al. (2001), pois, dos três sistemas comerciais para ajuste da vazão em função de alterações de velocidade, apenas um deles teve desempenho satisfatório, com tempos médios de 1,56 e $1,75 \mathrm{~s}$.

Para o sistema experimental de controle da rotação da bomba de pulverização desenvolvido 
por FIGUEIREDO \& ANTUNIASSI (2006), apesar da reprovação quanto ao tempo de resposta, pôde-se comprovar maiores tempos na fase crescente da troca de dosagem.

Os distribuidores centrífugos também demonstraram contrastes no experimento realizado porFULTON et al. (2005), pois, dentre 4 equipamentos comerciais, apenas um realizava a troca de dosagem em menos de 5s. No trabalho dos autores, também se destacaram os tempos de atraso, de $2 \mathrm{~s}$ até $-4,9 \mathrm{~s}$, que demonstrou que os equipamentos iniciaram a troca com atraso ou anteriormente ao ponto adequado. Esses dados demonstram um aspecto importante dos equipamentos à $\mathrm{TV}$, pois comprovam que o desempenho pode ser otimizado pela configuração mais correta dos tempos de adiantamento, visando a correção do tempo de atraso.

No trabalho de SCHUMANN et al. (2006), foi encontrado o sistema de controle mais rápido dentre todos analisados, capaz de realizar trocas de dosagem em menos de um segundo, com tempos de 0,31 a 0,46s. Apesar de o equipamento do ensaio 2 atender ao limite de 5 s, foi reprovado, pois, para a operação do tipo single-tree testada, foram exigidas transições em menos de um segundo. Entretanto, ambos os equipamentos comerciais realizaram transições mais rapidamente que os estudados por FULTON et al. (2005). MILLER et al. (2004) também validaram o equipamento para acionar e desligar a dosagem em função da aferição da presença de plantas. Os trabalhos realizados por MILLER et al. (2004), FULTON et al. (2005) e SCHUMANN et al. (2006) demonstram que os esforços dos projetos da Flórida, para melhoria da fertilização em citros, estão tendo resultados positivos, apesar de alguns equipamentos terem sido reprovados.

O tempo de adiantamento comprometeu o desempenho do equipamento comercial testado por MOLIN \& MENEGATTI (2006), pois, observandose os gráficos divulgados pelos autores, observa-se que os comandos de troca ocorreram antecipadamente aos pontos comandados pelo mapa de aplicação. Apesar do potencial de correção para esse aspecto, os tempos de resposta foram demasiados, próximos a 3 s para a fase crescente e 6 s para a fase decrescente. Já o equipamento experimental de CERRI (2001), apesar de apresentar tempos excessivos para o limite estabelecido, foi validado pelo autor para a aplicação estudada. Esse equipamento, apresentando tempos médios de 10,80 e $6,97 \mathrm{~s}$, respondeu positivamente à premissa de menores valores de tempo em fase decrescente.
Os dados de desempenho das máquinas à TV no regime estacionário confirmam a existência de equipamentos que operam próximo das metas e de outros com erros excessivos. No Brasil, uma semeadora à TV de milho, avaliada por HÖRBE (2012), desenvolveu a implantação da cultura com populações menores que as metas. No ensaio 1, a máquina operou em um limite de $3 \%$, proposto por CERRI (2001), enquanto, no ensaio 2, os erros ultrapassaram $12 \%$, o que foi atribuído a intempéries após a implantação da cultura. Já uma semeadora avaliada por FULTON (2006) apresentou desempenho insatisfatório e expressivamente inferior, pois os erros atingiram $-34,75 \%$, o planejamento da população de plantas ficaria no campo mais comprometido em relação à HÖRBE (2012) e, para esse nível de erro, o uso de uma máquina à TV não seria justificado, pois os benefícios buscados com a tecnologia não seriam atendidos.

Passando-se a analisar a acurácia dos equipamentos, correspondente aos regimes estacionários testados, verificou-se que, dentre os 13 equipamentos e 19 ensaios apresentados na tabela 2, apenas 6 apresentaram desempenho no limite de determinado por CERRI (2001), de 3\%, de acordo com os dados médios apresentados. Dentre estes, somente em um ensaio os valores máximos também estavam dentro do limite estipulado.

Dentre as semeadoras, o ensaio 1 de HÖRBE (2012) demonstrou alto potencial de acurácia para a máquina. Entretanto, o ensaio 2 apresentou desempenho inferior. Já a semeadora avaliada por FULTON (2006) não apresentou dados satisfatórios, cujo melhor desempenho foi de $-5,00 \%$.

Dentre os distribuidores centrífugos, o único equipamento que apresentou desempenho adequado em diferentes condições de dosagem foi o desenvolvido por CERRI (2001), que foi considerado aprovado pelo autor, apesar de observados valores máximos de $9,00 \%$. Os dados do autor demonstram que a magnitude da dosagem afetou a acurácia, afirmativa corroborada por FULTON et al. (2001), em que a menor dosagem teve desempenho inferior e não validada. Entretanto, os erros máximos do equipamento foram os maiores verificados, de até 78,04\%.

Além dos contrastes para as dosagens de um mesmo equipamento, FULTON et al. (2003) e MILLER ET AL. (2004), comprovaram que, assim como para os tempos de resposta, a acurácia apresentou contrastes expressivos entre os equipamentos comerciais. Em ambos os casos, somente um equipamento foi aprovado dentre três testados. No trabalho de FULTON et al. (2003), apesar do equipamento do ensaio 3 ter sido aprovado 
Tabela 2 - Acurácia de máquinas à taxa variável.

\begin{tabular}{|c|c|c|c|c|c|c|c|c|}
\hline \multirow{2}{*}{ Referência } & \multirow{2}{*}{ Ensaio } & \multicolumn{3}{|c|}{----------------Acurácia (\%)--------------- } & \multicolumn{3}{|c|}{------------------CV (\%)---------------. } & \multirow{2}{*}{$\begin{array}{l}\text { Metodologia } \\
\text { (tipo/avaliação) }\end{array}$} \\
\hline & & Mínima & Média & Máxima & Mínimo & Médio & Máximo & \\
\hline \multirow{2}{*}{$\mathrm{HÖRBE}^{1}$} & 1 & $-0,70$ & $-1,30$ & $-2,90$ & $n d^{2}$ & $n d^{2}$ & $n d^{2}$ & \multirow{2}{*}{ direta/plantas } \\
\hline & 2 & $-7,10$ & $-9,40$ & $-12,40$ & $11,25^{3}$ & 14,20 & 16,75 & \\
\hline FULTON $^{4}$ & 1 & $-5,00$ & $-21,40$ & $-34,75$ & $\mathrm{nd}^{2}$ & $n d^{2}$ & $\mathrm{nd}^{2}$ & direta/plantas \\
\hline \multirow{3}{*}{ CERRI $^{5}$} & 1 & $-4,00$ & 3,00 & 9,00 & $\mathrm{nd}^{2}$ & $6,46^{3}$ & $\mathrm{nd}^{2}$ & \multirow{3}{*}{ direta/pesagem } \\
\hline & 2 & $-0,70$ & 1,40 & 7,60 & $\mathrm{nd}^{2}$ & 5,70 & $n d^{2}$ & \\
\hline & 3 & $-1,30$ & $-2,90$ & $-4,60$ & $\mathrm{nd}^{2}$ & 1,73 & $\mathrm{nd}^{2}$ & \\
\hline \multirow{2}{*}{$\begin{array}{l}\text { FULTON } \\
\text { et al. }^{6}\end{array}$} & 1 & $\mathrm{nd}^{2}$ & 10,89 & 78,04 & $n d^{2}$ & $21,30^{3}$ & $\mathrm{nd}^{2}$ & \multirow{2}{*}{ direta/pesagem } \\
\hline & 2 & $\mathrm{nd}^{2}$ & 2,80 & 51,01 & $\mathrm{nd}^{2}$ & 20,00 & $\mathrm{nd}^{2}$ & \\
\hline \multirow{3}{*}{$\begin{array}{l}\text { MILLER } \\
\text { et al. }{ }^{7}\end{array}$} & 1 & $-1,00$ & 2,00 & 3,00 & $40,40^{8}$ & 47,90 & 55,40 & \multirow{3}{*}{ direta/pesagem } \\
\hline & 2 & 5,40 & 5,50 & 5,60 & 97,10 & 113,85 & 130,60 & \\
\hline & 3 & $-0,50$ & 10,43 & 20,70 & 17,20 & 40,13 & 53,60 & \\
\hline SERRANO et al. ${ }^{9}$ & 1 & 4,20 & 4,38 & 4,55 & $n d^{2}$ & $\mathrm{nd}^{2}$ & $\mathrm{nd}^{2}$ & direta/pesagem \\
\hline $\begin{array}{l}\text { MOLIN } \\
\text { MENEGATTI }^{10}\end{array}$ & 1 & $-18,40$ & $-20,79$ & $-26,60$ & $\mathrm{nd}^{2}$ & $\mathrm{nd}^{2}$ & $\mathrm{nd}^{2}$ & direta/pesagem \\
\hline \multirow{3}{*}{$\begin{array}{l}\text { FULTON } \\
\text { et al. }{ }^{11}\end{array}$} & 1 & $-1,43$ & $-4,26$ & $-5,89$ & $10,2^{3}$ & 11,78 & 13,00 & \multirow{3}{*}{ direta/pesagem } \\
\hline & 2 & $-3,21$ & $-4,50$ & $-5,27$ & 7,8 & 11,28 & 16,10 & \\
\hline & 3 & 0,27 & 2,59 & 4,29 & 5,8 & 17,27 & 27,40 & \\
\hline \multirow{3}{*}{ GARCIA $^{12}$} & 1 & 8,69 & 10,29 & 11,44 & $\mathrm{nd}^{2}$ & $\mathrm{nd}^{2}$ & $\mathrm{nd}^{2}$ & \multirow{3}{*}{ direta/pesagem } \\
\hline & 2 & 0,80 & 2,69 & 5,71 & $n d^{2}$ & $\mathrm{nd}^{2}$ & $n d^{2}$ & \\
\hline & 3 & 0,97 & 4,29 & 7,04 & $\mathrm{nd}^{2}$ & $\mathrm{nd}^{2}$ & $\mathrm{nd}^{2}$ & \\
\hline
\end{tabular}

${ }^{1}$ HÖRBE (2012): Médias de 50, 60, 70, 80 e 90mil sementes ha ${ }^{-1}$ de milho para cada ensaio.

${ }^{2}$ Dados não disponíveis ou não estimáveis, pelas informações divulgadas.

${ }^{3}$ Coeficiente de variação das variáveis observadas da acurácia.

${ }^{4}$ FULTON (2006): Média de 86,5; 123,6; 160,6 e 197,7mil sementes ha ${ }^{-1}$ de algodão.

${ }^{5}$ CERRI (2001): Equipamento centrífugo, dois discos, arrasto. Ensaios: 1) 2; 2) 3; 3) $5 \mathrm{Mg} \mathrm{ha}^{-1}$ de calcário.

${ }^{6}$ FULTON et al. (2001): Equipamento centrífugo, dois discos, veicular. Ensaios: 1) 56 e 2) $168 \mathrm{~kg} \mathrm{ha}^{-1}$.

${ }^{7}$ MILLER et al. (2004): Equipamentos para acionar e desligar a dosagem de $560 \mathrm{~kg} \mathrm{ha}^{-1}$ de calcário, pela presença de dossel vegetativo simulado de citros. Ensaios: 1) centrífugo, dois discos, com sensores fotocélulas; 2) pneumático, com sensores ultrassônicos; 3) centrífugo, dois discos, com sensores fotocélulas.

${ }^{8} \mathrm{CV}$ da distribuição transversal e longitudinal do insumo aplicado.

${ }^{9}$ SERRANO et al. (2007): Centrífugo, dois discos, acoplado. Média de 100 e $400 \mathrm{~kg} \mathrm{ha}^{-1}$ de superfosfato.

${ }^{10}$ MOLIN E MENEGATTI (2003): Centrífugo, dois discos. Média de 50,100, 150, 200 e $250 \mathrm{~kg} \mathrm{ha}^{-1}$ de ureia.

${ }^{11}$ FULTON et al. (2003): Médias de 3 a 4 dosagens, de 56 a $366 \mathrm{~kg} \mathrm{ha}^{-1}$ de cloreto de potássio. Ensaios: 1) equipamento pneumático, arrasto; 2 e 3) equipamentos centrífugos, dois discos, veiculares.

${ }^{12}$ GARCIA (2007): Dosador helicoidal de fertilizantes. Ensaios: 1) 50; 2) 100 e 3) $200 \mathrm{~kg} \mathrm{ha}^{-1}$.

pelas condições médias, apresentou erro de até $4,29 \%$. O equipamento do ensaio 1, de MILLER et al. (2004), foi aprovado também quando analisado o erro máximo, de 3,00\%. Entretanto, quando analisados os tempos testados para os mesmos equipamentos, todos haviam sido aprovados, demonstrando que, para validar as máquinas à $\mathrm{TV}$, não se deve analisar somente um dos critérios de desempenho separadamente.

Os demais ensaios para distribuidores, apresentados por SERRANO et al. (2007) e MOLIN E MENEGATTI (2003), não apresentaram acurácia dentro do limite especificado. Eles diferiram na magnitude e no sentido do erro. 
O equipamento experimental para controle de dosadores de fertilizantes, desenvolvido por GARCIA (2007), que havia sido aprovado nos tempos, quando avaliada a acurácia, apenas obteve aprovação para a média do ensaio 2. O pior desempenho na dosagem baixa confirma a premissa de que é uma taxa de pior desempenho para as máquinas à TV, sendo corroborado por FULTON et al. (2001) e CERRI (2001).

A acurácia, quanto à estabilidade da dosagem, medida pelo coeficiente de variação, pode ter um limite estipulado em $20 \%$, de acordo com FULTON et al. (2003). Os equipamentos avaliados pelos autores apresentaram coeficientes de variação adequados para as condições médias e inadequados para o valor máximo do ensaio 3 . Pode-se inferir que não houve relação direta entre a estabilidade e a magnitude do erro, pois o ensaio 3 foi o de melhor acurácia. Em FULTON et al. (2001), observaram-se valores próximos do limite especificado.

As melhores condições de estabilidade de dosagem para distribuidores foram encontradas por CERRI (2001), de até $6,46 \%$, enquanto que as condições menos adequadas foram verificadas em MILLER et al. (2004). Para todos os ensaios realizados por esses autores, a variabilidade ultrapassou os limites, atingindo valores maiores que $100 \%$. Entretanto, enquanto os demais dados da variabilidade tratam da variação da dose total do equipamento, para os autores, os valores representam a variabilidade de toda a área de aplicação, ou seja, a cada ponto coletado da matriz de coletores. Essa instabilidade ao longo da área de aplicação é um fator de erro expressivo e recorrente para os distribuidores centrífugos, e uma limitação para seu uso em TV.

Para as semeadoras, os dados disponibilizados por HÖRBE (2012) demonstram que elas podem operar com variabilidades intermediárias aos distribuidores.

\section{CONCLUSÃo}

Os sistemas de controle atualmente utilizados na TV estão disseminando-se rapidamente em diferentes intervenções agrícolas, necessitando-se esclarecer seu desempenho, pois é isso que garante a efetividade do manejo localizado. A partir das informações apresentadas, foi possível identificar quais aspectos estão envolvidos no desempenho das máquinas e quais metodologias podem ser consideradas para sua avaliação. Os níveis de desempenho descritos mostram um cenário preocupante, com reprovação de $59 \%$ dos equipamentos para tempos de resposta e 53\% para acurácia. O desempenho das máquinas demonstrou contrastes expressivos para cada operação agrícola. Os tempos de resposta tiveram a maior taxa de reprovação para pulverizadores, $67 \%$, seguido dos distribuidores, não validados em $56 \%$ dos casos. Quanto à acurácia, foram observados também contrastes entre magnitudes de dosagem para um mesmo equipamento. Os distribuidores foram reprovados quanto à acurácia em $60 \%$ dos casos, enquanto que as semeadoras em $50 \%$. Os sistemas experimentais, que passaram por métodos de aperfeiçoamento, apresentaram melhor desempenho nas avaliações. As informações apresentadas demonstram que as máquinas podem comprometer severamente o manejo localizado e os resultados da AP e da TV.

\section{REFERÊNCIAS}

ANDRADE-SANCHEZ, P.; HEUN, J. Understanding technical terms and acronyms used in Precision Agriculture. Arizona Cooperative Extension, Tucson, 2010. Disponível em: $<$ http:// arizona.openrepository.com/arizona/handle/10150/146427>. Acesso em: 05 mar. 2012.

ANTUNIASSI, U.R. et al. Performance evaluation of injection metering systems. Revista Brasileira de Engenharia Agrícola e Ambiental, v.6, n.1, p.159-165, 2002.

ASABE (AMERICAN SOCIETY OF AGRICULTURAL AND BIOLOGICAL ENGINEERS). ASAE S341.3. Procedure for measuring distribution uniformity and calibrating granular broadcast spreaders. St. Joseph, 2004. 5p.

ASABE (AMERICAN SOCIETY OF AGRICULTURAL AND BIOLOGICAL ENGINEERS). Standardization procedures. Disponível em $<$ http://www.asabe.org/media/52623/projects.pdf $>$. Acesso em: 19 mar. 2012

CERRI, D.G.P. Desenvolvimento de um sistema de aplicação localizada de calcário a taxas variáveis. 2001. 90f. Dissertação (Mestrado em Agronomia) - Universidade de São Paulo, São Paulo.

FIGUEIREDO, Z.N.; ANTUNIASSI, U.R. Uso de controladores lógico programáveis para ensaios de avaliação de sistemas de controle eletrônico para pulverizadores. Revista Energia na Agricultura, Botucatu, v.21, n.2, p.134-146, 2006.

FULTON, J.P. et al. Performance assessment and model development of a variable-rate, spinner-disc fertilizer applicator. Transactions of ASAE, St. Joseph, v.44, n.5, p.1.071-1.081, 2001.

FULTON, J.P. et al. Comparison of variable-rate granular application equipment. In: ASAE ANNUAL MEETING, 2003, Las Vegas. Proceedings... Las Vegas: ASAE, 2003, p.1-15.

FULTON, J.P. et al. Rate response assessment from various granular VRT applicators. Transactions of ASAE, St. Joseph, v.48, n.6, p.2095-2103, 2005.

FULTON, J.P. et al. Evaluation of variable-rate seeding for cotton. In: PROJECT REPORT, COTTON COMMISSION, 
2006. Disponível em <http://www.aaes.auburn.edu/com/pubs/ researchreports/08cottonrr.pdf $>$. Acesso em: 28 mar. 2011.

GADANHA JUNIOR, C.D. et al. Avaliação do tempo de resposta de controladores eletrônicos de pulverização em função da variação da velocidade. In: SIMPÓSIO INTERNACIONAL DE TECNOLOGIA DE APLICAÇÃO DE AGROTÓXICOS, 2., 2001, Jundiaí, SP. Anais... Jundiaí: SBEA, 2001, p.1-7.

GARCIA, A.P. Desenvolvimento de um sistema de controle eletro-mecânico para dosador de fertilizantes. 2007. $120 \mathrm{f}$. Dissertação (Mestrado em Engenharia Agrícola) - Universidade Estadual de Campinas, Campinas, SP.

HÖRBE, T. de A.N. Agricultura por ambiente: manejo sítio específico da população de milho. 2012. 58f. Dissertação (Mestrado em Ciência do Solo) - Universidade Federal de Santa Maria, Santa Maria, 2012.

MILLER, W.M. et al. Evaluating variable rate granular fertilizer technologies in Florida citrus. Proceedings of Florida State Horticultural Society, Lake Alfred, v.117, p.161-166, 2004.

MOLIN, J.P. Agricultura de precisão. Parte 2: diagnóstico, aplicação localizada e considerações econômicas. Engenharia Agrícola, Jaboticabal, v.17, n.2, p.108-121, 1997.
MOLIN, J.P.; MENEGATTI, L.A.A. Desempenho de distribuidora a lanço com doses variáveis de uréia. Engenharia Agrícola, Jaboticabal, v.23, n.2, p.290-299, 2003.

OGATA Engenharia de controle moderno. 5.ed. São Paulo: Saraiva, 2011. 824p.

SCHUMANN, A.W. et al. Variable rate granular fertilizations of citrus groves. Applied Engineering in Agriculture, St. Joseph, v.22, n.1, p.19-24, 2006.

SERRANO, J.M. et al. Avaliação de um distribuidor centrífugo de adubo na perspectiva de utilização em agricultura de precisão. Revista de Ciências Agrárias, Lisboa, v.30, n.1, p.79-87, 2007.

SHEARER, S.A. et al. Considerations for development of variable-rate controller test standard. In: ASAE ANNUAL INTERNATIONAL MEETING, 2002, Chicago. Anais... Chicago: ASAE e CGIR, 2002, p.1-12.

UMEZU, C.K. Sistema de controle de um equipamento de formulação, dosagem e aplicação de fertilizantes sólidos a taxas variáveis. 2003. 171f. Tese (Doutorado em Engenharia Agrícola) - Universidade Estadual de Campinas, Campinas, SP. 